\title{
Technological Progress in Radiation Therapy for Brain
}

\section{Tumors}

\section{Frederik Jozef Vernimmen, Kathy Rock}

Department of Radiation Oncology, Cork University Hospital, Cork, Ireland. Email: Frederik.Vernimmen@hse.ie

Received November $6^{\text {th }}, 2013$; revised December $5^{\text {th }}, 2013$; accepted December $13^{\text {th }}, 2013$

Copyright (C) 2014 Frederik Jozef Vernimmen, Kathy Rock. This is an open access article distributed under the Creative Commons Attribution License, which permits unrestricted use, distribution, and reproduction in any medium, provided the original work is properly cited. In accordance of the Creative Commons Attribution License all Copyrights (C) 2014 are reserved for SCIRP and the owner of the intellectual property Frederik Jozef Vernimmen, Kathy Rock. All Copyright (C 2014 are guarded by law and by SCIRP as a guardian.

\section{ABSTRACT}

To achieve a good therapeutic ratio the radiation dose to the tumor should be as high as possible with the lowest possible dose to the surrounding normal tissue. This is especially the case for brain tumors. Technological advancements in diagnostic imaging, dose calculations, and radiation delivery systems, combined with a better understanding of the pathophysiology of brain tumors have led to improvements in the therapeutic results. The widely used technology of delivering 3-D conformal therapy with photon beams (gamma rays) produced by Linear Accelerators has progressed into the use of Intensity modulated radiation therapy (IMRT). Particle beams have been used for several decades for radiotherapy because of their favorable depth dose characteristics. The introduction of clinically dedicated proton beam therapy facilities has improved the access for cancer patients to this treatment. Proton therapy is of particular interest for pediatric malignancies. These technical improvements are further enhanced by the evolution in tumor physiology imaging which allows for improved delineation of the tumor. This in turn opens the potential to adjust the radiation dose to maximize the radiobiological effects. The advances in both imaging and radiation therapy delivery will be discussed.

\section{KEYWORDS}

\section{Brain Tumors; Radiotherapy; Proton Therapy; Functional Imaging}

\section{Introduction}

Shortly after the discovery of x-rays in 1895, irradiation also became used as a therapeutic modality. However the application of these radiation treatments was hampered by a lack of understanding of the radiobiology of ionizing radiation and technical factors such as lack of tissue penetration due to the low energy of the photons and inadequate information on the dose distribution in and around the tumor.

Over the decades radiation has always played a role in managing brain tumors either as the primary modality or as adjuvant therapy. Technological advancements in diagnostic imaging, radiation delivery systems, and a better understanding of the radiobiology have consolidated and expanded this role.

Modern radiotherapy depends on good quality imaging to define the target and surrounding organs at risk (OAR), rapid and accurate dose calculations by treatment planning systems (TPS), equipment to deliver these complicated treatment plans, and imaging to verify the correctness of the radiation delivered. The purpose of this paper is to discuss recent technological advances in these areas, discuss the clinical benefit and to look at future developments.

\section{Radiation Therapy}

Irradiation is the deposition of energy (dose) in the target by various radiation modalities using a variety of irradiation techniques. This dose is expressed in units of Gray (Gy), and the beam energy used to deliver the dose is expressed as Mega Volts (MV). It is the absorption of this energy by the cell structures that causes the individ- 
ual cell damage resulting in control of the disease.

Gamma (photon) radiation can be delivered by a number of different radiation therapy machines. The most widely used for brain tumors is the Linear Accelerator (Linac). Very few departments still have or use Cobalt machines. Linear accelerators produce photon beams of variable energies (6 - $20 \mathrm{MV}$ ) by accelerating electrons and steering them onto a metal target. This interaction produces the primary photon beam which is then modified for each individual patient Figure 1. Other machines such as the Gamma knife ${ }^{\circledR}$ and Cyberknife ${ }^{\circledR}$ also use gamma rays to deliver the therapeutic dose, but their application is mainly in stereotactic radiosurgery for benign lesions and brain metastasis. The basic application of Linacs is by 3-D conformal therapy, whereby a number of individually shaped beams produced in the gantry are directed at an isocenter, which is a fixed geometric point in the treatment room and which can be placed in the centre of the target volume by moving the couch onto which the patient is positioned. This 3-D conformal therapy has been the worldwide standard for many years.

Delivery of the therapeutic dose can also be achieved by using charged particle beams such as negative pimesons, helium and carbon ions and protons. A particle beam is produced by a cyclotron or a synchrotron and they all have particular dose distribution characteristics (Bragg peak) which make it theoretically a better radiation modality in and around sensitive structures such as the brain as there is no dose distal to the Bragg peak and a lower dose proximal to the target Figure 2. In addition some of these particles have also a radiobiological advantage over photon beams in that they cause more radiobiological damage per unit of dose. In other words their Radiobiological Effect (RBE) is higher (1). Protons are the most commonly available and are used by way of double scattering the primary narrow beam to a beam of

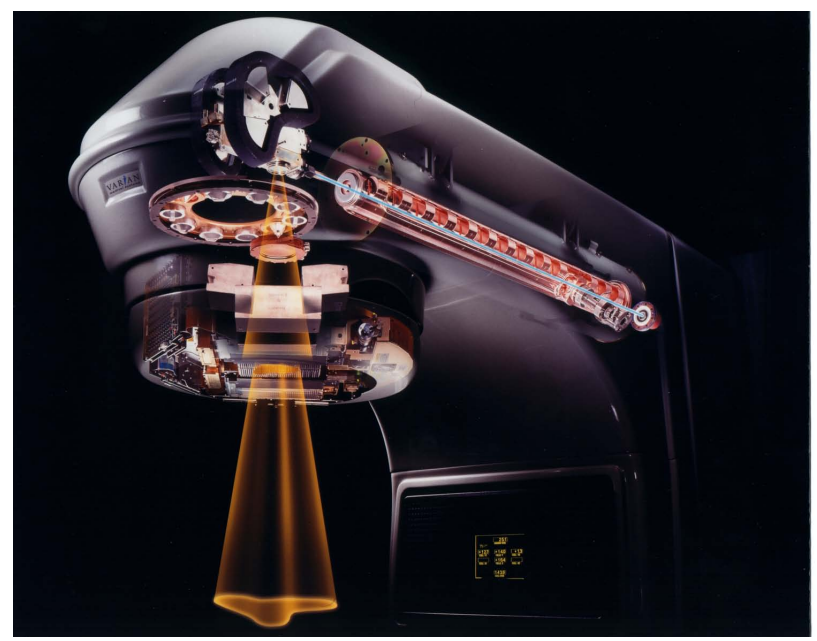

Figure 1. Diagram of a Linear Accelerator showing the electron acceleration (blue) and the photon beam (yellow).



Figure 2. Diagram showing the brag peak and SOPB compared to a $10 \mathrm{MV}$ photon beam.

sufficient size for clinical use. To cover the target volume the Bragg peak needs to be spread out over the full thickness. This dose delivery technique is referred to as a Spread Out Bragg Peak (SOBP) and has been the standard use since protons were introduced clinically. The availability of proton therapy facilities worldwide is still limited. The availability of other charged particle beams, such as carbon ions and helium ions are even more limited.

Negative pi-mesons have been investigated for their potential in the treatment of Glioblastoma Multiforme $[1,2]$ on the basis of their radiobiological advantage in the area of the Bragg peak. However they need very powerful cyclotrons (500 MeV) to be produced and clinical studies had to use cyclotrons in physics research laboratories. This was not a suitable clinical environment for patients. Negative pi-mesons are no longer in clinical use.

\section{Technological Advances in Gamma Radiationtherapy}

The conventional 3D technique is more and more being superseded by the use of Intensity Modulated Radiotherapy (IMRT). This technique also uses a number of beams, but the energy fluency throughout each individual beam can be modulated by the use of a number of small individually movable leaves. IMRT is evolving fast due to improvements in the acceleration process of the electrons, allowing for faster beam cycling, and significant progress in the manipulation of the beam once produced. Latest versions of Linacs have omitted the beam flattening filter allowing for higher dose rates, reduced energy variation across the beam, and reduced leakage $[3,4]$. The number of leaves have been increased with a corresponding decrease in the size of each individual leaf 
allowing for very conformal dose profile. The speed with which the individual leaves move and their position verification during the delivery of the radiation has also improved [5]. All this allows for rapid beam cycling, improves the geometry of the fluency in the beam and further enhances the benefits of IMRT. These advances have led to the more recent use of Volumetric Arc Therapy (VMAT) [6]. During this treatment delivery process, a beam with a rapidly changing energy fluency profile is rotated 360 degrees around the patient, delivering the overall treatment much faster compared to the use of separate individual beams.

To verify the position of the patient prior to each treatment session many Linacs have now additional equipment built in for On Board Imaging (OBI). This consists of a flat panel detector and either uses a separate $\mathrm{KV}$ source or the primary beam for cone beam ct scanning of the patient in the treatment position.

The delivery of such high quality beams can also be done under stereotactic conditions due to improved mechanical accuracy of the rotation of gantry and couch around the isocenter with an accuracy of $\leq 1 \mathrm{~mm}$. This combined with improved patient immobilization devices and position verification using the on board imaging, as described above, allows for the delivery of fractionated stereotactic radiotherapy (FSRT).

\section{Technological Advances in Particle Radiation Therapy}

The main focus for improvements has been for proton beams. The number of facilities is continuously growing worldwide making this treatment more readily available for patients. This availability has been further enhanced by the development of small cyclotrons and smaller treatment delivery systems, allowing these proton machines to be installed in areas not much bigger than the standard area for a Linac [7-9]. This reduces the cost and makes them more of a proposition for smaller hospital facilities. Treatment delivery is moving towards spot scanning, a technique that uses the thin primary proton beam without inherent beam modifications. This eliminates the neutron contamination that is present in double scattered beams used up to now and allows for intensity modulated proton therapy (IMPT) by controlling the motion and energy of the spot [10]. Patient robotic positioning systems and image verification systems are on par with the latest developments in Linac therapy. The dose verification of IMPT treatments is difficult and challenging but has already become routine practice in some of the proton therapy centers [11].

\section{Technological Advances in Diagnostic Imaging}

Radiotherapy for brain tumors has benefited enormously from the technical progress in CT scanning, MRI scanning as well as PET scanning. This benefit is further enhanced by the ability of modern treatment planning systems to integrate all this information into the planning systems, providing the radiation oncologist with maximum information for target delineation.

Conventional MR imaging has been used for many years in the diagnosis of primary brain tumors but up to now has only given pure anatomical information.

Advances in scanning speed, better spatial resolution, and improvement in hardware and 3D algorithms have all impacted on radiotherapy delivery. Functional imaging techniques such as Diffusion Weighted Imaging (DWI), Dynamic contrast-enhanced MRI (DCE MRI), Proton MR Spectroscopy and Perfusion Weighted Imaging have all helped in the determination of the tumor extent.

Diffusion Weighted Imaging (DWI) reflects the cell density of tissue and can potentially indicate areas with a higher tumor burden, but interpretation of the typical diffusion-weighted imaging features of primary neoplasm is variable. The apparent diffusion coefficients (ADC) of high grade gliomas have been shown to be lower than that of low grade gliomas but ADC maps alone are insufficient for predicting type and grade of glial neoplasms. Dynamic Contrast-Enhanced MRI reflects permeability of the microvasculature and blood flow which is correlated to the oxygenation of the tumor, which in turn has an impact on the tumor's radiosensitivity.

Diffusion tensor imaging can delineate more accurately the macroscopic tumor versus the infiltrating component of the tumor within the peritumoural edema and the normal brain parenchyma [12].

Dose painting is the prescription of a deliberate nonuniform dose distribution to a target volume based on functional or molecular imaging identifying areas of increased radio resistance. This is a novel paradigm in radiation oncology.

Dose painted targets are imaging surrogates for areas associated with poor radio responsiveness. Partial volume boosting involves the selection of a "target within a target" based on information gained from molecular imaging. This allows for a higher dose to be delivered to identified areas within the target that are most resistant while sparing other surrounding normal tissue [13].

Fluorine-18 (18F) fluorodeoxyglucose (FDG) positron emission tomography (PET) is limited in its evaluation of brain tumors due to the high basal activity of the white matter and cerebral cortex. Other radioactive tracer isotopes have also been investigated. For the treatment of craniopharyngioma in the pediatric population carbon-11 methionine PET has been shown to have a significantly greater uptake within the tumor compared with non-in- 
volved white matter making it more useful then FDG PET in identifying active tumor [14]. Carbon-11 methionine PET has also been demonstrated to be useful in the delineation of meningiomas [15].

\section{Clinical Benefits}

Very few tumors are truly radio resistant and could actually be destroyed if sufficiently high radiation doses could be administered safely. Advancements in tumor imaging has led to improved target delineation this in turn can allow the dose to be "sculpted" resulting in a better therapeutic ratio. As mentioned above the progress in imaging and the ability to import that information into TPS platforms has not only allowed for a more accurate delineation of the anatomy of the target and OARs but also the ability to import tumor physiological information. Modern TPS platforms can very quickly calculate the IMRT dose distribution in and around the target. Thanks to advanced software the visual interpretation of these dose distributions has been enhanced. At the same time dose distributions can also be presented in a numerical way allowing checking for dose-volume tolerances/constraints and comparison of treatment plans. All this has clinical benefit because it allows giving a higher radiation dose to the target whilst better sparing the Oar's. Individual IMRT administration in its early applications was time consuming with an associated reduction of patient capacity per Linac. The introduction of VMAT has shortened these delivery times considerably which provides for greater patient comfort. There is less likelihood of patient movement during treatment and greater overall patient capacity per Linac. The favorable dose distribution from IMRT opens the way to give the overall total radiation dose in a smaller number of fractions, called hypofractionation. Instead of using the more conventional scheme of 30 - 35 fractions over 6 - 7 weeks, a hypofractionation of 10 sessions in 2 weeks for treating Glioblastoma multiforme becomes feasible [16]. This has significant advantages for the patient as it reduces the number of hospital visits, and it improves quality of life for patients with aggressive brain tumors who have a limited prognosis [16]. Although re-irradiation of a tumor for recurrence is fraught with dangers, IMRT allows a bit more leeway when considering re-treatments for recurrence.

The ability of OBI systems to regularly verify target positioning and tumor volume change, combined with the capabilities of modern TPS for rapid recalculation of dose distribution plans allows for compensation of these changes, the so called adaptive radiotherapy approach. This can be done without interrupting the normal treatment course and has obvious benefits in terms of target coverage and dose to OAR.

With regard to the clinical benefit of proton therapy compared to IMRT there is ongoing debate on this, especially in the light of the high cost of proton facilities. Nevertheless, although equally high target doses can be achieved by both modalities, the lower integral dose and the stopping power of protons give it an advantage in terms of normal tissue radiation toxicity. This lower toxicity is of particular importance in patients with brain tumors and children. Children have the most pronounced benefit in terms of their developing organs that might otherwise be irradiated and in terms of late carcinogenic effects $[17,18]$. Hence proton therapy is very attractive to treat this young population group and the body of literature covering this is rapidly growing [19-21].

The specific dose profile of protons makes them also ideal modalities for re-treatments and for intracranial stereotactic therapy [22-25].

In terms of patient positioning and imaging the progress has been on par with developments in photon therapy. Practically all new proton facilities have robotic positioning systems as a standard part of the equipment.

\section{Future Developments}

Unless a new way of accelerating electrons is used, the basic functioning of the Linac will remain, but further advances in the beam delivery can be expected. Imaging during the treatment with its associated potential for positioning corrections is improving rapidly, and real time tracking of organ and target motion during therapy will become more and more common with more and more centers having equipment to do this. This capability will expand as the manufacturers incorporate this technology into standard equipment. This imaging will not necessarily remain in the domain of $\mathrm{x}$-rays but will also include MRI real time imaging [26] and PET scanning. This will open the way to not only adjust for anatomical changes but also to adapt for physiological changes within the tumor during the course of the radiation treatment.

New ways of accelerating protons to clinically useful energies have been explored. A considerable amount of research is done on laser plasma acceleration [27] which has the promise of offering a small overall treatment unit easily accommodated in a radiotherapy department. A similar development of a small machine is the Dielectrical wall accelerator (DWA) [28], which although working on a different acceleration principle could be easily accommodated in a limited space.

\section{Conclusion}

Radiotherapy for brain tumours has seen impressive technological progress providing better tumour delineation on the planning systems, better and more accurate dose distributions, and better control of important parameters during the duration of a radiotherapy course. This in turn 
allows for better results and improved quality of life for the patients.

\section{REFERENCES}

[1] G. Goodman, P. Dixon, J. Bowen, C. Gaffney and M. Pomeroy, "Pi-Meson Radiotherapy at TRIUMF," Journal of Gastroenterology, Vol. 12, No. 2, 1990, pp. 85-99.

[2] G. Schmitt, C. F. von Essen, R. Greiner and H. Blattmann, "Review of the SIN and Los Alamos Pion Trials," Radiation Research Supplement, Vol. 8, 1985, pp. S272-S278. http://dx.doi.org/10.2307/3576657

[3] P. Tsiamas, J. Seco, Z. Han, M. Bhagwat and J. Maddox, "A Modification of Flattening Filter Free Linac for IMRT,” Medical Physics, Vol. 38, No. 5, 2011, pp. 23422356. http://dx.doi.org/10.1118/1.3571419

[4] D. Georg, T. Knoos and B. McClean, "Current Status and Future Perspective of Flattening Filter Free Photon Beams,” Medical Physics, Vol. 38, No. 3, 2011, pp. 12801293. http://dx.doi.org/10.1118/1.3554643

[5] G. A. Davies, G. Poludniowski and S. Webb, "MLC Tracking for Elekta VMAT: A Modelling Study,” Physics in Medicine and Biology, Vol. 56, No. 23, 2011, pp. 7541-7554. http://dx.doi.org/10.1088/0031-9155/56/23/013

[6] Bedford JL, Thomas MD, Smyth G. "Beam Modeling and VMAT Performance with the Agility 160-Leaf Multileaf Collimator," Journal of Applied Clinical Medical Physics, Vol. 14, No. 2, 2013, pp. 4136-4142.

[7] Editors, "Mevion Proton Therapy System Approved in US, Cleared for Firing up at Initial Hospitals MedGadget," 2012.

http://www.medgadget.com/2012/06/Mevion-proton-ther apy-system

[8] Newsfeed, "IBA to Install ProteusOne Proton Therapy System in Taiwan,” Medical Physics Web, 2013. http://medicalphysicsweb.org/cws/article/newsfeed/53919

[9] Newsfeed, "MIT, Protom Team up on Proton Therapy," Medical Physics Web, 2008. http://medicalphysicsweb.org/cws/article/research/35751

[10] R. Mohan, A. Mahajan and B. D. Minsky, "New Strategies in Radiation Therapy: Exploiting the Full Potential of Protons," Clinical Cancer Research, Vol. 19, No. 23, 2013, pp. 6338-6343. http://dx.doi.org/10.1158/1078-0432.CCR-13-0614

[11] L. Archambault, F. Poenisch, N. Sahoo, D. Robertson and A. Lee, "Verification of Proton Range, Position, and Intensity in IMPT with a 3D Liquid Scintillator Detector System,” Medical Physics, Vol. 39, No. 3, 2012, pp. 1239-1246. http://dx.doi.org/10.1118/1.3681948

[12] D. A. Sanghvi, "Recent Advances in Imaging of Brain Tumors,” Indian Journal of Cancer, Vol. 46, No. 2, 2009, pp. 82-87. http://dx.doi.org/10.4103/0019-509X.49145

[13] S. M. Bentzen and V. Gregoire, "Molecular ImagingBased Dose Painting: A Novel Paradigm for Radiation Therapy Prescription,” Seminars in Radiation Oncology, Vol. 21, No. 2, 2011, pp. 101-110. http://dx.doi.org/10.1016/j.semradonc.2010.10.001
[14] B. S. Laser, T. E. Merchant, D. J. Indelicato, et al., "Evaluation of Children with Craniopharyngioma Using Carbon-11 Methionine PET Prior to Proton Therapy,” Neuro-Oncology, Vol. 15, No. 4, 2013, pp. 506-510. http://dx.doi.org/10.1093/neuonc/nos321

[15] L. Götz, T. S. Spehl, W. A. Weber and A. L. Q. Grosu, "PET and SPECT for Radiation Treatment Planning," Quarterly Journal of Nuclear Medicine and Molecular Imaging, Vol. 56, No. 2, 2012, pp. 163-172.

[16] K. Reddy, D. Damek, L. E. Gaspar, D. Ney and A. Waziri, "Phase II Trial of Hypofractionated IMRT with Temozolomide for Patients with Newly Diagnosed Glioblastoma Multiforme," International Journal of Radiation Oncology, Biology, Physics, Vol. 84, No. 3, 2012, pp. 655-660.

[17] E. R. Dennis, M. R. Bussiere, A. Niemerko, M. W. Lu and B. C. Fullerton, "A Comparison of Critical Structure Dose and Toxicity Risks in Patients with Low Grade Gliomas Treated with IMRT versus Proton Radiation Therapy," Technology in Cancer Research and Treatment, Vol. 12, No. 1, 2013, pp. 1-9.

[18] B. S. Athar and H. Paganetti, "Comparison of Second Cancer Risk Due to Out-of-Field Doses from 6-MV IMRT and Proton Therapy Based on 6 Pediatric Treatment Plans,” Radiotherapy \& Oncology, Vol. 98, No. 1, 2011, pp. 87-92.

http://dx.doi.org/10.1016/j.radonc.2010.11.003

[19] B. J. Moeller, M. Chintagumpala, J. J. Philip, D. R. Grosshanss and M. F. McAleer, "Low Early Ototoxicity Rates for Pediatric Medulloblastoma Patients Treated with Proton Radiotherapy,” Radiation Oncology, Vol. 6, 2011, p. 58. http://dx.doi.org/10.1186/1748-717X-6-58

[20] G. Suneja, P. D. Poorvu, C. Hill-Kayser and R. A. Lustig, "Acute Toxicity of Proton Beam Radiation for Pediatric Central Nervous System Malignancies,” Pediatric Blood \& Cancer, Vol. 60, No. 9, 2013, pp. 1431-1436. http://dx.doi.org/10.1002/pbc.24554

[21] H. Hauswald, S. Rieken, S. Ecker, K. A. Kessel and K. Herfarth, "First Experiences in Treatment of Low Grade Glioma Grade I and II with Proton Therapy,” Radiation Oncology, Vol. 7, 2012, p. 189.

http://dx.doi.org/10.1186/1748-717X-7-189

[22] M. Mizumoto, T. Okumura, E. Ishikawa, T. Yamamoto and S. Takano, "Reirradiation for Recurrent Malignant Brain Tumor with Radiotherapy or Proton Beam Therapy. Technical Considerations Based on Experience at a Single Institution,” Strahlentherapie und Onkologie, Vol. 189, No. 8, 2013, pp. 656-663. http://dx.doi.org/10.1007/s00066-013-0390-6

[23] M. W. McDonald, M. R. Wolanski, J. W. Simmons and J. C. Buschbaum, "Technique for Sparing Previously Irradiated Critical Normal Structures in Salvage Proton Craniospinal Irradiation,” Radiation Oncology, Vol. 8, 2013, p. 14. http://dx.doi.org/10.1186/1748-717X-8-14

[24] F. Vernimmen, J. K. Harris and J. A. Wilson, "Stereotactic Proton Beam Therapy of Skullbase Meningiomas," International Journal of Radiation Oncology, Biology, Physics, Vol. 99, No. 1, 2001, pp. 99-105. http://dx.doi.org/10.1016/S0360-3016(00)01457-7

[25] F. J. Vernimmen, Z. Mohamed, J. Slabbert and J. Wilson, 
"Long Term Results of Stereotactic Proton Beam Radiotherapy for Acoustic Neuromas," Radiotherapy and Oncology, Vol. 90, No. 2, 2009, pp. 208-212. http://dx.doi.org/10.1016/j.radonc.2008.11.004

[26] T. Bierre, S. Crijns, P. M. Rosenschold, M. Aznar and L. Specht, "Three Dimensional MRI-Linac Intra-Fraction Guidance Using Multiple Orthogonal Cine-MRI Planes,” Physics in Medicine and Biology, Vol. 58, No. 14, 2013, pp. 4943-4950.

http://dx.doi.org/10.1088/0031-9155/58/14/4943
[27] S. Kawata, T. Izumiyama, T. Nagashima, M. Takano and D. Barada, "Laser Ion Acceleration toward Future Ion Beam Cancer Therapy-Numerical Simulation Study,” Laser Therapy, Vol. 22, No. 2, 2013, pp. 103-114. http://dx.doi.org/10.5978/islsm.13-OR-09

[28] G. J. Caporaso, T. R. Mackie, S. Sampayan and Y. J. Chen, "A Compact Linac for Intensity Modulated Proton Therapy Based on a Dielectric Wall Accelerator," Physica Medica, Vol. 24, No. 2, 2008, pp. 98-101. http://dx.doi.org/10.1016/j.ejmp.2008.01.010 